\title{
Use of Photo Diary to Explore Needs for Digital Disease Management Program among Community Older Adults with Chronic Disease
}

\author{
Kuo MC ${ }^{1}$, Chen $\mathrm{CM}^{2 *}$, $\mathrm{Wu} \mathrm{FG}^{3}$, Chen $\mathrm{CH}^{4}$, Yin $\mathrm{ZX}^{5}$ and Wang $\mathrm{CY}^{6}$ \\ ${ }^{1}$ Assistant Professor, Department of Nursing, Cardinal Tien Junior College of Healthcare and \\ Management, Taiwan \\ ${ }^{2}$ Professor, Department of Nursing / Graduate Institute of Gerontology, College of Medicine, \\ National Cheng Kung University, Taiwan \\ ${ }^{3}$ Professor, Department of Industrial Design, National Cheng Kung University, Taiwan \\ Research Article \\ Volume 4 Issue 2 \\ Received Date: April 09, 2020 \\ Published Date: April 30, 2020 \\ DOI: $10.23880 /$ eoij-16000235 \\ ${ }^{5}$ Associate Professor, Department of Computer Science and Information, Southern Taiwan University of Science and Technology, \\ Taiwan \\ ${ }^{6}$ Assistant, Department of Nursing, National Cheng Kung University, Taiwan
}

*Corresponding author: Professor Chen Ching-Min, Department of Nursing / Graduate Institute of Gerontology, College of Medicine, National Cheng Kung University, No. 1, University Rd., East Dist., Tainan City 70101, Taiwan, Tel: +88662353535*5858; Email: chingmin@mail.ncku.edu.tw

\section{Abstract}

As technology advanced, new e-health solutions are evolved to empower people to manage their care at home. This study explored the needs for disease management in activity tracking using photo diary through older adults' subjective perspective. It further aimed to suggest which lifestyle measures, symptoms and behaviors would be meaningful to include in such a digital diseases care management program for technology design. Both photo diary and focus group discussion were used, 11 older adults with multiple metabolism-related chronic diseases (Mean age, $72.5 \pm 6.14$ years) were recruited and asked to carry out the photo diary to trace their living situation and needs using a tablet camera. A focus group discussion was applied to identify the needs of chronic disease management, basing on the results of living context tracing. Five themes, regular physical activity, health management, and healthy diet, regular daily routine and social connection, were identified by content analysis from photo diary and the focus group discussion. The results indicated that the photo diary program can raise awareness and promotes positive behavior changes. It is believed that the E-approach can be applied to the effectively enhance older adults' self-management by monitoring their health status and their daily routine activities.

Keywords: Living Context; Disease Care Management; Photo Diary

\section{Introduction}

Chronic diseases are common debilitating which are the main causes of poor health, disability, and premature death [1]. In the US, around half (50.9\%) of adults have at least one chronic condition and $26 \%$ have two or more 
conditions from the 2010 National Health Interview Survey [2]. With no exception, the prevalence of multiple chronic conditions among older Taiwanese adults was increased as the worldwide population ages. Nearly half of all older adults in Taiwan are living with at least one chronic health condition (a relative increase of $52.5 \%, 42.3 \%$ in 2000 and $64.5 \%$ in 2010) [3]. The coexistence of 2 or more chronic conditions has become widely prevalent, with the hypertension and type 2 diabetes mellitus as the most common diseases across all older populations [4]. The burden of chronic diseases continues to grow globally, and the majority of the older population will face advanced illness which makes general health and functioning declined in the near future.

The vital direction for enhancing well-being and health care for older adults is to better manage their conditions [5]. Adherence to chronic disease management is critical to achieving improved health outcomes. Global strategies for chronic diseases might be possible by targeting common risk factors such as healthy lifestyle [1]. In reality, there is no known curative treatment for chronic disease as its various and complex causes. It is increasingly important to understand patient's own efforts on control of chronic diseases through self-management [6]. Interventions targeting chronic diseases have focused on the changes in through self-management strategies [7].

There is a general agreement that the person with the diseases is the manager of a chronic illness [8]. The ability of the patient to control the chronic conditions is significantly influenced by behavioral factors [9]. Facilitating patients' self-management may be the best way to reduce the chronic disease burden and the need for care, as the effective self -management strategies will yield healthy life extension [8].

High-quality chronic diseases self-management behaviors have been shown to improve patient disease control [10]. The elder population should be prioritized for the reinforcement of behaviors and lifestyle modification to control their chronic conditions. However, it has shown that primary chronic diseases care poor link to the older person's real home, where healthy behaviors that influence chronic disease occur. Living context is an essential component to offer the older person and healthcare professionals important information about chronic disease self-management in which daily health decisions about food, activity, medication use, and other healthy behaviors occur [11]. Commonly, the living contexts for health control are very minimally known in detail in primary care settings in which the dynamics of the older person-disease relationship are essentially invisible. Circadian activity rhythm analysis learns older adults' activity patterns and feeds the older person back into the behaviors can be added to context-aware power management [12].
The photo diary is a qualitative research method in which the researcher gives the interviewee a list of prompts for taking photos. After taking the photos the researcher interviews the participants, using the photos to elicit information. This method brings the participants' images and thereby gives multiple dimensions to their perspective. It can be used to gather a multi-faceted understanding of participants' lives. The method is useful to understand episodic events or experiences in more detail. Mobile technologies are increasing, which initiatives to use for supporting self-management includes tracing their physical condition and their living context [13]. They can record the rhythmic behavioral activity of older adults and detects changes within these patterns. Clinical behavioral patterns of older adults will be extracted from real data sets, and behavioral changes were studied by older adults and health professions. Patients and healthcare providers can investigate any difficulty in compliance by targeting on the region of the anomaly as identified by living context. These typically require participants to document their experiences through a series of diary entries. The method may later be used to prompt discussion with the users. Photographs can be incorporated into diary entries to enrich the data. They also ease the burden on older adults to capture and later recall their contents [14].

Self-management strategies can facilitate adherence to these lifestyle changes. Assuming that increased in older adult participation is a possible approach, we design a self-report method with tracing their living situation and needs using a tablet camera to understand self-management situation of chronic diseases. To gain relevant aspects of chronic disease management, subjects were asked to participate in focus group discussions to include storytelling by reading photos together retrieved data on their experiences, opinions and perspectives. The program aimed to explore relevant aspects of self-management with chronic diseases, for use in the development of a digital diseases care management program. It further aimed to suggest which lifestyle measures, symptoms and behaviors would be meaningful to be included in such a program.

\section{Material and Methods}

\section{Design}

The qualitative method including photo diary and focus group discussion were used to comprehend subjects' disease care management needs

\section{Sample and Procedures}

The subjects of this study were the older people recruited from one community in southern Taiwan. Eligible 
participants were older adults (aged 65 years and over) with more than one of the metabolism related diseases, include hypertension, diabetes or hyperlipidemia as there were the most common diseases observed among Taiwanese older adults. We excluded older people who were not able to communicate, had impaired cognitive function, and had hearing problems. Prior to data collection, subjects were invited to participate in the photo diary training program. Eligible participants were informed of study protocols, then sign an informed consent form. When the eligible participants enrolled, personal data would be collected. After two weeks training, participants were invited to carry out daily photo diary voluntarily for lasting three months. They traced their living situation such as activities and rest, dieting situation, and taking medicine and individual or environment support needs for chronic disease management using a camera with a tablet. Thereafter, the researcher contacted them weekly to ask whether they had questions regarding tablet operation and collect the photographs during the course. Finally, we used a focus group discussion to ensure the needs of chronic disease management, basing on the results of living context tracing and measurements.

\section{Focus Group Question Guide}

According to the recommended focus group methodology [15], focus group questions were developed by the researcher from the content analysis of a photo diary, aiming to identify needs influencing self-management behaviors for chronic diseases. The question guide consisted of five subthemes including regular physical activity, health management, and healthy diet, regular daily routine and social connection which allowed participants to get acquainted and feel connected, and to start the discussion of the topic. The key questions were used to guide the group towards the main part of the discussion and to focus on the purpose of this study. Finally, older adults were asked to share ideas concerning self-management promotion as well as e-health intervention strategies to counter unhealthy behaviors in aging populations.

The focus group lasted 2 hours and was facilitated by the researchers as moderator and facilitator. The moderator guided the group towards the main part of the discussion and to target on the purpose of this study. During the focus group discussion, the moderator followed the results of a photo diary, but asked side opinions to obtain more in-depth information about the topics, and showed enough flexibility to allow open discussions between older adults. The method is useful to understand the experiences about chronic diseases self-management behaviors in more detail. Clinical behavioral patterns of older adults were extracted from real data sets.

\section{Data Analysis}

Content analysis was used for qualitative data analysis. Researchers read and analyzed each photograph from tablets at least twice to identify similarities, differences, and general patterns. All photographs related to chronic disease management were extracted. Then were organized into clusters that reflected similar behaviors. Identified and validated categories were then discussed with research team. Together, the researcher engaged in analysis and synthesis to identify emerging categories, which grouped that, reflected the behaviors of chronic disease management. To ensure reliability of data interpretations, doubts or disagreements were discussed with the other researcher until consensus was reached. Four criteria of credibility, transferability, dependability, and confirmability were used to evaluate study rigor and trustworthiness [16]. The researcher participated in the same activities with participants in community centers to establish good relationships that would encourage their free recording of tracing living context related to chronic disease management. The researcher immediately lists the photography systematically and discussed findings with the other authors to achieve credibility. Open-ended focus group discussion was used to verify participant responses and asked participants to validate their findings to establish transferability. To ensure data dependability, the first author only took responsibility for collecting data. A detailed review, data reconfirmation, and the recruitment of older adults who were willing to share experiences established confirmability.

\section{Results}

\section{Sample Characteristics}

From December 2015 to March 2016, 11 participants were recruited and completed the photo diary program, 7 of those subjects also involved in focus group discussion. Table 1 showed the demographic characteristics of the participants. The mean age of all subjects was 72.45 (range from 67 to 87) years. There were more female represented in this study. All participants lived with their families. The two most common chronic diseases were hypertension and type 2 diabetes mellitus. Table 2 showed demographic data of seven participants, which took part in the focus group discussion for identifying the needs of chronic disease management based on the results of living context tracing and measurements. Half participants had more than one chronic medical condition. 


\begin{tabular}{|c|c|c|c|c|c|c|}
\hline \multirow[b]{2}{*}{ Variable } & \multicolumn{2}{|c|}{ Total subjects $(n=11)$} & \multicolumn{2}{|c|}{ focus group participant $(n=7)$} & \multicolumn{2}{|c|}{$\begin{array}{l}\text { focus group non-participant } \\
\qquad(n=4)\end{array}$} \\
\hline & $\mathbf{N}$ & $\%$ & $\mathbf{N}$ & $\%$ & $\mathbf{N}$ & $\%$ \\
\hline Age & $\begin{array}{c}72.45 \pm \\
6.14\end{array}$ & & $74.29 \pm 7.70$ & & $69.25 \pm 2.63$ & \\
\hline \multicolumn{7}{|c|}{ Gender } \\
\hline Male & 3 & 27.3 & 2 & 28.6 & 1 & 25 \\
\hline Female & 8 & 72.7 & 5 & 71.4 & 3 & 75 \\
\hline \multicolumn{7}{|c|}{ Marital } \\
\hline Without spouse & 1 & 9.1 & 1 & 14.3 & 0 & 0 \\
\hline With spouse & 10 & 90.9 & 6 & 85.7 & 4 & 100 \\
\hline \multicolumn{7}{|c|}{ Education } \\
\hline No degree & 1 & 9.1 & 1 & 14.3 & 0 & 0 \\
\hline Junior high degree or less & 9 & 81.8 & 6 & 85.7 & 3 & 75 \\
\hline $\begin{array}{c}\text { Senior high degree or } \\
\text { above }\end{array}$ & 1 & 9.1 & 0 & 0 & 1 & 25 \\
\hline \multicolumn{7}{|c|}{ Religion } \\
\hline None & 1 & 9.1 & 0 & 0 & 1 & 25 \\
\hline Taoist, Buddhist & 9 & 81.8 & 7 & 100 & 2 & 50 \\
\hline Catholic, Christianity & 1 & 9.1 & 0 & 0 & 1 & 25 \\
\hline \multicolumn{7}{|c|}{ Living with Family } \\
\hline Yes & 11 & 100 & 7 & 100 & 4 & 100 \\
\hline No & 0 & 0 & 0 & 0 & 0 & 0 \\
\hline \multicolumn{7}{|c|}{ Diseases } \\
\hline cardiavascular disease & 1 & 9.1 & 1 & 14.3 & 0 & 0 \\
\hline type 2 diabetes mellitus & 6 & 54.5 & 4 & 57.1 & 2 & 50 \\
\hline hypertension & 9 & 81.8 & 6 & 85.7 & 3 & 75 \\
\hline
\end{tabular}

Table 1: Descriptive Information for the Sample ( $\mathrm{N}=11)$.

\begin{tabular}{|c|c|c|c|}
\hline Case & Age (years) & Gender & Comorbidity \\
\hline \multirow{2}{*}{ A } & \multirow{2}{*}{72} & \multirow{2}{*}{ Male } & 1. Hypertension \\
\hline & & & 2. Hyperlipidemia \\
\hline B & 83 & Male & Hypertension \\
\hline $\mathrm{C}$ & 66 & Female & Diabetes \\
\hline \multirow{2}{*}{$\mathrm{D}$} & \multirow{2}{*}{69} & \multirow{2}{*}{ Female } & 1. Diabetes \\
\hline & & & 2. Arrhythmia \\
\hline$E$ & 72 & Female & Hypertension \\
\hline \multirow{2}{*}{$\mathrm{F}$} & \multirow{2}{*}{71} & \multirow{2}{*}{ Female } & 1. Hypertension \\
\hline & & & 2. Diabetes \\
\hline \multirow{2}{*}{ G } & \multirow{2}{*}{71} & \multirow{2}{*}{ Female } & 1. Hypertension \\
\hline & & & 2. Hyperlipidemia \\
\hline
\end{tabular}

Table 2: Demographic Characteristics (focus group discussion). 


\section{Five Subthemes}

Five subthemes (regular physical activity, health management, and healthy diet, regular daily routine and social connection) were identified by content analysis of a photo diary. Focus group discussion were used to induce subjects' expressed self-management behaviors for chronic diseases based on the results of living context tracing through photo diary.

\section{Regular Physical Activity}

Physical activities generally refer to a movement that enhances health. Subjects expressed that everyone performs physical activity in order to promote health. Walking was as a mode of both exercise and transportation. Most subjects went out regularly in the morning or in the evening based on photographs represented. Walking was the physical activity of choice across all subjects. The places they went usually were nearby home, the park, and the temple as shown with the photo taken. Some participants chose to stretch their bodies by themselves or using the exercise equipment in the park. During the focus group discussion, Case B said, "The exercise equipment in the park would be a great benefit to the chronic disease control." But Case A said, "To promote and maintain my health, I would not follow the recommendation that the expert suggested. We required modification of physical activity for environmental factors such as weather. When the weather rained or was too hot, I just walked around my house." Several participants reported that they went out to neighborhood regularly; it is notable that older adults, used "walking" as mode of physical activity showed it in the photographs and the focus group discussion. We found that accessibility of facilities and outdoor fitness equipment was associated with physical activity. Poor weather was also a barrier to physical activity.

\section{Health Management}

Implementation of health management for the older adults has the potential for significant impacts on the improvements in the health of the older people. It could help older adults to copy with a treatment plan for a health condition. Most subjects complied with the prescription including time and dosage as shown with a photo taken. Some participants took dietary supplements to provide nutrients. We also found that only two subjects regularly went back the medical clinics for follow up during the data collection period in focus group discussion, Case E said, "I seldom measured blood pressure before. After I began taking the photographs by the tablet for my living context, I measure my blood pressure every day." Case B said, "I measured my blood pressure twice a day, which were in the morning and the evening. I found that there were distinct data of the blood pressure at different time. I regularly went to the clinic and visited physician once a month. He gave me antihypertensive drugs. I know that when I feel dizzy, I must go to the hospital right away." Case C said, "I almost went the same hospital for the chronic diseases control. All my information was there." Case A said, "Sometimes I change the antihypertensive drugs based on the reading on blood pressure. If the data on blood pressure was low, I just took half dosage of antihypertensive drugs. I know that it was wrong because I am not a physician. I think that being long illed makes the patient a good doctor." Case G said, "I almost wake up around at the same time, five o'clock. Then I walked to the community center and measured blood sugar." Case F said, "When I got the drugs from the clinic, I put all drugs into the storage box. I could clearly remember when and whether I would take medicine."

Although multiple participants expressed the importance of compliance for medical regiment the prescription, only two participant's return for their checkup regularly. Accurate measurement of blood pressure and blood glucose are essential for the treatment of chronic diseases. Some subjects check their blood pressure or blood glucose regularly. It is good to know that some subjects began to take monitoring regularly after the photo diary. In summary, the photo diary program can promote positive behavior change. These methods apply to the maintenance of health care for older adults by monitoring their health status and their daily routine activities in their own environment. Adequate applications can lead older adults to make sense of the changes they are doing in their daily life.

\section{Healthy Diet}

Healthy diet is an important part of health behavior as it plays a substantial role to delay the progress of their chronic diseases and postpone death. In this study, we found that some older people also take the dietary supplements. But few older adults took the high calories or low nutrition food such as bread, cake and milk tea. Older people living alone would lunch and eat dinner outside. If they cooked for themselves, they usually ate the leftover from previous meals for consecutive days. We saw that some older people ate more meat and fish, but less vegetables and fruit from photographs. Some older people living with family took unhealthy food, such as fried or pickled food. During the focus group discussion, Case B said, "We must control foodintake, because the aged-related organ and physiological function degeneration would cause longstanding dyspepsia." Case G said, "I cook today, but it is difficult for me to control the portions of food. I must eat the same food for more meals." Case B, Case F and Case E also had the same situation. Case C said, "Although I cook for my families, I prepared their most favorite food. But I seldom cook fish, because of bad smell." Case B said, "I usually prepare meat for my Youngers. But I ate less meat." Case D said, "I seldom cook for myself. Most of the 
time my son cooks for me and his son. I usually had the meal outside with the child. " But few older people would not like to have the meal outside. Case B said, "restaurant food usually contain additional substance, it would be harmful for health. The food cooking by myself will be better and clean. " Case $\mathrm{C}$, case D and case F had the same opinion. But case B said, "Although I did not like to have the meals outside, my families usually had the dinner outside. If we did not go out with them, it was so strange."

In this study, older adults were found to eat less fruits and vegetables and more leftover from previous meals. Most of the time, the food was prepared outside of the home. They seldom cook for themselves. Loneliness can contribute to inadequate food intakes. Food choice is motivated by individual attributes that mediated by familial factor. Poor diet quality is known to increase the risk for chronic disease.

\section{Regular Daily Routine}

Among the common problems related to aging is sleep quality. Changes in circadian sleep-wake patterns are associated with poor sleep. Maintenance of routine may be particularly adaptive in older adults. Routine lifestyle rhythms may include the number of times per day and the duration of time for eating meals, watching television, shopping, cleaning, exercise, or social engagements. In our study as shown from the photo taken, most subjects keep regular hours in basic activities, such as meal time, sleep time, wake time, physical activity patterns. Some older people took a nap every afternoon. Changing lifestyle rhythms happened due to tracing living contexts. Case E said, "I stay up late. Almost one o'clock!! After tracing my living context, I went to sleep earlier than before."

Regular lifestyles with orchestrated physiological circadian rhythms lead to prevent common disease. Needless to say, modern sleep habits, such as later sleep and wake up times, disturb the regular light-dark cycle. Thus, the regular sleep-wake rhythm is critical. By self-managing circadian rhythm it would assist to establish the suitable lifestyle for individuals.

\section{Social Connection}

The older people were particularly vulnerable to perceive unusual stress such as the loss of relatives, retirement, and the deterioration of physical function. The social connection affects the older person's physical and emotional wellbeing. When older adults face the risk of illness, and serious life event, social support may be especially important. In our study community-based care centers regularly hold weekly activities and some tour activities for older adults. Furthermore, some older adults got news from newspaper, television, and cell phone to avoid social dislocation. Case B said, "The Lizhang (chief of the village) encourages us to go out for participating activities." Case G said, "There were various courses in our community. The chief of village encouraged us to engage in leisure-time activities. If we had the classes, we could not feel very sleepy all day. Supposing I stayed at home, I would sleep day long. Weakness disappeared when you walked a lot." Case G said, "We are easy to forget what we learn, such as using tablet for tracing our living context. We ask each other how to operate. We are close to each other" Loneliness is negatively related to emotional well-being among older adults. These activities could enhance social interaction.

\section{Discussion}

Chronic diseases are the main causes of poor health, disability, and death, and account for most of health care expenditures. The diseases themselves can be potentially managed with appropriate lifestyle modifications. Diseases such as type II diabetes, cardiovascular disease and the metabolic syndrome are closely related and often stem from the same risk factors. Treatment for most chronic diseases involves lifestyle modifications to slow or reverse disease progression. In this study, hypertension and type 2 diabetes mellitus were the two most common chronic diseases. This result is similar to Kuo, Jeng, Chen, and Jian [4]. These two diseases were the most frequently reported age- and sexspecific diagnoses for multiple physician visits among older patients in Taiwan. They are also the chronic diseases that contribute to a significant portion of the health care expenditure for a nation as individuals with diabetes and hypertension need continuous medical care [17].

The chronic disease burden has largely resulted from risk factors including poor diet, physical inactivity, bad habits, and uncontrolled physiological index (such as blood pressure and blood sugar) [18]. Proactive solutions to help manage chronic disease are an important issue for most health care systems. Enhancing health promotion and self-management of chronic disease, it is possible to maintain age-related illness in a better condition. In our study, the healthy habits of chronic diseases consist of five major components from the contents by the photo diary taken and the focus group discussion: (1) regular physical activity; (2) health selfmanagement; (3) healthy diet; (4) regular routine activities; and (5) social connection. Summary of main findings and our recommendations are listed in Table 3. Most older adults walked out home nearby, the park, and the temple. They used the exercise equipment to stretch their bodies in the park. They also participated in various courses provided by community-based care center. These physical activities are to promote older adults' physical and emotional well-being. Strong evidence shows that physical inactivity increases the risk of many adverse health conditions, including coronary 
heart disease, type 2 diabetes, shortens life expectancy [19]. A Taiwanese study found that compared with individuals who were inactive had a $26 \%$ lower mortality risk [20]. As is the older adult for chronic disease, the health benefits of physical activity surpass those of conventional medications [21]. The modern technology now allows everyone to easily capture photographs. To design appropriate technological solutions, it is crucial to understand the specific barriers to mobilize older adults from their perspectives. Development of the physical activity record system is has been strongly recommended in this study. The use of physical activity record application systems has been an easy method for collecting self-reported physical activity data. The data can be used to perform a behavior analysis. Physical activity record system allows older adults to autonomously document their experiences to support generation of older adult's needs and requirements. Chronic disease management often requires a long-term care plan. Adherence to chronic disease management is critical to achieving improved health outcomes. Patients' nonadherence to prescribed medications is associated with poor therapeutic outcomes and progression of disease [22]. In this study, most of the older adults complied with the prescription including time and dosage. But one subject took half of antihypertensive drug off by himself, basing on the readings of his blood pressure. The patients with chronic disease are the principal caregiver and in the latter an active producer, so they must become partners of the physician. The patient must understand and assent to new responsibilities. Taking the patient's views into account was associated with better compliance, and greater continuity of care. In the future, the effective and efficient methods of management will be developed that enhance the ability of patients with chronic disease to participate in their health care. The first is self-management education that addresses the medication adherence. An important element for patients is learning from each other, and the principal reason for the benefit is growing in confidence in their ability to cope with their disease. Development of a drug information application software and the push notification service are recommended. To schedule a medication and the dosage confirmation, then an announcement of each prescription is made via the push notification service. This application finally gets older adults to take their medicine.

\begin{tabular}{|c|l|c|}
\hline Main Finding & \multicolumn{1}{|c|}{ Interview Statement and Photo Diary Shown } & Strategy \\
\hline Regular physical activity & $\begin{array}{l}\text { Went out regularly in the morning or in the evening Walking was } \\
\text { the choice of physical activity } \\
\text { "To promote and maintain my health, I would not follow the } \\
\text { recommendation that the expert suggested." }\end{array}$ & $\begin{array}{c}\text { Development of physical } \\
\text { activity record }\end{array}$ \\
\hline Health management & $\begin{array}{l}\text { Most subjects complied with the prescription including time and } \\
\text { dosage } \\
\text { Some participants took dietary supplements } \\
\text { Only two subjects regularly went back the medical clinics } \\
\text { "I seldom measured blood pressure before. After I began taking } \\
\text { the photographs by the tablet for my living context, I measure my } \\
\text { blood pressure every day." } \\
\text { "I measured my blood pressure twice a day, which were in the } \\
\text { morning and the evening." } \\
\text { "Sometimes I change the antihypertensive drugs basing the data } \\
\text { on blood pressure. I know that it was wrong because I am not a } \\
\text { physician." }\end{array}$ & $\begin{array}{c}\text { Development of a drug } \\
\text { information application } \\
\text { software and the push } \\
\text { notification service }\end{array}$ \\
\hline Healthy diet & $\begin{array}{l}\text { Few older adults took the high calories or low nutrition food } \\
\text { Most elderly living alone ate lunch and dinner outside } \\
\text { If they cook for themselves, they usually ate the leftover from } \\
\text { previous meals. } \\
\text { "I cook today, but it is difficult for me to control the portions of } \\
\text { food. I must eat the same food for more meals." } \\
\text { "I usually prepare meat for my youngers." }\end{array}$ & $\begin{array}{c}\text { Exploitation of interactive } \\
\text { nutrition games }\end{array}$ \\
\hline
\end{tabular}




\begin{tabular}{|c|l|l|l|}
\hline Regular daily routine & $\begin{array}{l}\text { Most subjects keep regular hours in basic } \\
\text { activities Some elderly took a nap every afternoon } \\
\text { "I stay up late. Almost one o'clock!! After tracing my living context, } \\
\text { I went to sleep earlier than before." }\end{array}$ & $\begin{array}{c}\text { Development of a reminder } \\
\text { system }\end{array}$ \\
\hline Social connection & $\begin{array}{l}\text { Community-based care centers regularly hold weekly } \\
\text { activities and some tourist activities for older adults } \\
\text { Some older adults got news from newspaper, } \\
\text { television, and cell phone to avoid social dislocation } \\
\text { "There were various courses in our community. The chiefof village } \\
\text { encouraged us to engage in leisure-time activities." }\end{array}$ & $\begin{array}{l}\text { Development of online } \\
\text { community interaction }\end{array}$ \\
\hline
\end{tabular}

Table 3: Summary of main findings and recommendations.

Dieting is a major lifestyle-related risk factor of chronic diseases. With regard to major chronic diseases (including type 2 diabetes and cardiovascular diseases), the most often mentioned dietary recommendation are whole-grain products, a high intake of fruits, vegetables, and a low intake of red or processed meat [23]. Dietary supplements are regularly used by the adult population, which are intended to supplement the normal diet. People who use dietary supplement are more likely than nonusers to adopt a number of positive health-related habits, including better dietary patterns, exercising regularly, and maintaining a healthy body weight. Dietary supplement users are more likely than nonusers to pay attention to their diets and to make an effort to improve dietary intake [24]. Not only is the intake of certain nutrients associated with an increased health risk, high-caloric intake also plays a crucial role. It is important to assess individual long-term dietary intake. Exploitation of interactive nutrition games is recommended by the study participants. Interactive games and tools will teach older adults about healthy eating. Sufficiently engaging games might enhance the effectiveness of health messaging, allowing people to practice useful thought patterns and behaviors and encouraging them to learn from mistakes in safe virtual environments. The disease itself can be potentially avoided or successfully managed with appropriate lifestyle modifications [25]. Efficient and effective sleep is critical for the proper function of many organ systems. Disturbances of both sleep and the underlying circadian rhythms have long been associated with many diseases [26]. However, circadian timekeeping abnormalities are characteristic of the ageing process. Sleep can be improved with a healthy approach to the lifestyle and a structured approach to sleep hygiene [27]. In this study subjects positively changed circadian rhythms after the photo diary program through a reminder system.

Both social isolation and loneliness are associated with increased mortality and impair quality of life and well-being. Social isolation is a particular problem at older ages. Socially isolated individuals are at increased risk for the development of chronic disease and psychiatric disease [28]. Lee, $\mathrm{Wu}$,
Chang, Weng, Wu, \& Chen [19] indicated that higher levels of social interaction appeared to help suppress depression in old age and maintain the physical functions of senior adults. Participation in social groups should therefore be strongly recommended for promoting the health of older adults. In our study community-bases care centers regularly hold activities and encouraged older adults' proactive participation. Reducing both social isolation and loneliness are important for quality of life and well-being for older adults. Technology helps older adults keep in touch with families, bringing new stimuli into their lives and providing more access to information. The technology can increase social interaction and maintain engagement during life. The community is no longer easily defined as a physical place, but is a set of social relationships. Development of online community interaction is recommended. We can try to give older adults the possibility to share their experiences on social networks, perhaps with others who have the same diseases who can provide suggestions and emotional support. Technology can help them to keep in touch with families, friends when they live alone.

Ory, et al. [8] indicated that self-management can change health behaviors, improve health status, and reduce health care utilization and costs. The most serious challenges for self-management are empowering and engaging patients with chronic diseases to manage their own health therapeutic programs. Patients with chronic diseases empowerment reflects the ability of patients to positively influence their health and health behavior. The expansion of activity tracking and personal data collection offers the potential for patient engagement in the management of chronic diseases. In our study some subjects began to change health behavior such as blood pressure monitoring and sleep earlier. And some subjects monitor physiological index of chronic diseases after the photo diary program. The information also offers health care providers real world assessments of their patients' daily activity patterns. Health care supported by mobile devices is a potentially highimpact tool to improve health outcomes among those living 
with chronic diseases through enhanced chronic disease management adherence. Electronic health has become an important tool for healthcare systems. Further development and evaluation of self-management information systems for chronic diseases will be critical, especially how these tools overcome barriers to chronic disease management. More innovation and optimization research in a living context, tracing information systems has the potential to transform the promise of mobile health technologies into the reality of improved health care delivery and outcomes. We believe that with the development of information technology, integrating living contexts to facilitate health into the technology platform may be effectively used by more and more patients.

\section{Limitation}

Our study has several potential limitations. First, most of our participants who used the tablet tracing their living context were women. Gender differences in the behaviors of older adults with chronic diseases were ignored. Second, although 11 older adults were invited to carry out the photo program, living context regarding chronic diseases during the data collection period was not totally taken into account. Third, our study consisted of only older adults from Taiwan, which limited the generalization of findings to other older populations. Fourth, our study does not capture all the relevant aspects of living context with chronic disease profiles tracing by the tablet. Despite these limitations, our study provides the influence from tracing living context. In the further information systems successfully implemented to support care for chronic illness is critical.

\section{Authors Contribution}

Conceptualization, C.M.C. and F.G.W.; formal analysis, C.Y.W.; methodology,C.M.C., F.G.W., C.H.C. and Z.X.Y.; project administration, C.M.C.; writing-original draft, M.C.K.; writing - review and editing, C.M.C. All authors have read and agreed to the published verson of the manuscript.

\section{Funding}

This work was supported by Ministry of Science and Technology (grant number: MOST 104-2420-H-006 019MY3).

\section{Conflict of Interest}

The authors have no conflict of interests to declare.

\section{References}

1. Bauer UE, Briss PA, Goodman RA, Bowman BA (2014) Prevention of chronic disease in the 21st century: elimination of the leading preventable causes of premature death and disability in the USA. Lancet 384(9937): 45-52.

2. Ward BW, Schiller JS (2013) Prevalence of multiple chronic conditions among US adults: estimates from the National Health Interview Survey, 2010. Prev Chronic Dis 10: E65.

3. Fu S, Huang N, Chou Y (2014) Trends in the Prevalence of Multiple Chronic Conditions in Taiwan From 2000 to 2010: A Population-Based Study. Prev Chronic Dis 11: 140205.

4. Kuo MC, Jeng C, Chen CM, Jian WS (2013) Profile of elderly with multiple physician visits: Advocacy for tailored comprehensive geriatric assessment use in clinics. Geriatri Gerontol Int 14(2): 372-380.

5. Rowe JW, Fulmer T, Fried L (2016) Preparing for Better Health and Health Care for an Aging Population. JAMA 316: 1643-1644.

6. Ward BW, Schiller JS, Goodman RA (2012) Multiple Chronic Conditions Among US Adults: A 2012 Update. Prev Chronic Dis 11: E62.

7. Salive ME (2013) Multimorbidity in older adults. Epidemiol Rev 35: 75-83.

8. Ory MG, Ahn S, Jiang L, Smith ML, Ritter PL, et al. (2013) Successes of a national study of the chronic disease selfmanagement program: Meeting the triple aim of health care reform. Med Care 51(11): 992-998.

9. Longtin Y, Sax H, Leape LL, Sheridan SE, Donaldson L, et al. (2010) Patient participation: current knowledge and applicability to patient safety. Mayo Clin Proc 85(1): 5362.

10. American Diabetes Association (2015) Standards of medical care in diabetes-2015 abridged for primary care providers. Clin Diabetes 33(2): 97-111.

11. Qualls SH (2016) Caregiving families within the longterm services and support system for older adults. American Psychologist 71(4): 283-293.

12. Wood A, Stankovic JA, Virone G (2008) Context-aware wireless sensor networks for assisted living and residential monitoring. IEEE Network 22: 26-33.

13. Phillips RL, Short A, Dugdale P, Nugus P, Greenfield D (2014) Supporting patients to self-manage chronic disease: clinicians' perspectives and current practices. Aust J Prim Healt 20(3): 257-265. 
14. Carter S, Mankoff J (2005) When participants do the capturing: the role of media in diary studies. Poster session presented at the 23rd SIGCHI conference of the Association for Computing M.

15. Krueger RA (1998) Developing Questions for Focus Groups. Thousand Oaks, California: Sage Publications.

16. Lincoln YS, Guba EG (1999) Establishing trustworthiness. In: Bryman A, Burgess R (Eds.), Qualitative research, Sage, London, Englansd 3: 397-444.

17. Yang SH, Chen SF, Nieh S, Liu CL, Lin YS (2017) The Effect of Individual and Neighbourhood Socioeconomic Status on Diabetes Mellitus Survival in Working Age Patients in Taiwan. PLOS ONE 12(1): e0169550.

18. Boutayeb A, Boutayeb S (2005) The burden of non communicable diseases in developing countries. Int J Equity in Health 4(1): 2.

19. Lee KL, Wu CH, Chang CI, Weng LJ, Wu YC, et al. (2015) Active Engagement in Social Groups as a Predictor for Mental and Physical Health among Taiwanese Older Adults: A 4-year Longitudinal Study. Int J Gerontol 9: 1-6.

20. Wen CP, Wai JP, Tsai MK, Yang YC, Cheng TY, et al. (2011) Minimum amount of physical activity for reduced mortality and extended life expectancy: a prospective cohort study. Lancet 378(9798): 1244-1253.

21. Durstine JL, Gordon B, Wang Z, Luo X (2013) Chronic disease and the link to physical activity. J Sport Health Sci 2(1): 3-11.

22. DiMatteo MR (2004) Variations in patients' adherence to medical recommendations: a quantitative review of 50 years of research. Med Care 42(3): 200-209.

23. Boeing H, Bechthold A, Bub A, Ellinger S, Haller D, et al. (2012) Critical review: vegetables and fruit in the prevention of chronic diseases. Eur J Nutr 51(6): 637663.

24. Dickinson A, MacKay D (2014) Health habits and other characteristics of dietary supplement users: a review. Nutr J 13: 14.

25. Roberts CK, Barnard RJ (2005) Effects of exercise and diet on chronic disease. J Appl Physiol 98(1): 3-30.

26. Musiek ES, Xiong DD, Holtzman DM (2015) Sleep, circadian rhythms, and the pathogenesis of Alzheimer Disease. Exp Mol Med 47: e148.

27. Nishinoue N, Takano T, Kaku A, Eto R, Kato N, et al. (2012) Effects of sleep hygiene education and behavioral therapy on sleep quality of whitecollar workers: a randomized controlled trial. Ind Health 50(2): 123-131.

28. Barth J, Schneider S, von Känel R (2010) Lack of social support in the etiology and the prognosis of coronary heart disease: A systematic review and meta-analysis. Psychosom Med 72(3): 229-238. 